\title{
Lattice supersymmetry with Hopf algebra for the link approach
}

\author{
A. D'Adda, \\ Dipartimento di Fisica Teorica, Universita' di Torino and INFN Sezione di Torino \\ I-10125 Torino, Italy \\ E-mail: daddade.infn.it \\ N. Kawamoto and J. Saito* \\ Department of Physics, Hokkaido University \\ Sapporo, 060-0810 Japan \\ E-mail: kawamoto@particle.sci.hokudai.ac.jp \\ E-mail: saitodparticle.sci.hokudai.ac.jp
}

\begin{abstract}
A formalism of lattice supersymmetry based on a lattice-deformed superalgebra which was originally introduced in the link approach formulation is presented. We propose that the superalgebra can in fact be identified as a Hopf algebra, showing all the Hopf algebra axioms and consistencies are satisfied with explicit formulae. In particular, the "deformed" Leibniz rules proposed in the original link approach are now built in the coproduct structure of the Hopf algebra. Fields in this scheme, as representations of the Hopf algebra, are found to obey a kind of mildly deformed statistics, which is interpreted as a braiding strucutre. We can then construct, at least perturbatively, the corresponding lattice field theory, which has the Hopf algebraic symmetry with the deformed statistics, as an example of braided quantumm field theory formulated by Oeckl.
\end{abstract}

The XXVII International Symposium on Lattice Field Theory

July 26-31, 2009

Peking University, Beijing, China

\footnotetext{
* Speaker.
} 


\section{Introduction}

Regularization of supersymmetric field theory has increased its importance both for recent theoretical and phenomenological developments, including, for instance, gauge/gravity duals such as AdS/CFT and supersymmetry breaking. One may expect that lattice formulation, among others, would provide a promising regularization scheme with the applicability to strong-coupling, thus constructive and nonperturbative, analysis in the first principle calculation. It is, however, far from straightforward to incorporate supersymmetry on the lattice due to the discrete nature of spacetime; superalgebra, which prescribes supersymmetry, contains momentum operator, and momentum operator should be the generator of infinitesimal spacetime translation, which is broken on the lattice. For this difficulty, various approaches have been developed so far. (For a review see [U] and references therein.) In this article, we present a possible formulation [వ] of lattice supersymmetry with a "deformed" notion of superalgebra in the framework of the link approach [B], 团]. This deformation can be naturally interpreted as a generalization of Lie algebra to Hopf algebra. What we need to formulate is then a field theory with this Hopf algebraic supersymmetry. We show that such a formulation would be given by applying a general formalism called braided quantum field theory (BQFT) [[]]. For this purpose, we introduce a simply generalized statistics of fields which is compatible with the structure of our Hopf algebra. Supersymmetry on the lattice can now be recognized as various sets of Ward-Takahashi identities derived by this BQFT formalism [可].

We will illustrate these aspects in the following, mainly concentrating on two-dimensional non-gauge examples.

\section{Superalgebra in the link approach}

In the link approach [3] , superalgebra on a two-dimensional lattice was introduced in the form

$$
\left\{Q, Q_{\mu}\right\}=i \partial_{+\mu}, \quad\left\{\tilde{Q}, Q_{\mu}\right\}=-i \varepsilon_{\mu \nu} \partial_{-v},
$$

with the other commutators just vanishing. Notice that supercharges $Q_{A}=Q, Q_{\mu}$ and $\tilde{Q}$ are expressed in the Dirac-Kähler twisted basis, which essentially corresponds to $\mathscr{N}=(2,2)$ supercharges in two dimensions in the normal basis. ${ }^{1}$ We can see that fermions in the link approach should be geometrically distributed on the lattice just like the Dirac-Kähler or staggered fermions, where the d.o.f. of possible doublers on the lattice is essentially used as that of extended supersymmetry through the Dirac-Kähler twisting. This is why the twisted basis was chosen in the superalgebra above. Another point is that the algebra (‥J) contains the forward and backward finite difference operators $\partial_{ \pm \mu}$ which simply replace the momentum operator in the continuum. These difference operators don't obey the Leibniz rule, but obey the modified Leibniz rule

$$
\partial_{ \pm \mu}\left(\varphi_{1} \cdot \varphi_{2}\right)(x)=\partial_{ \pm \mu} \varphi_{1}(x) \varphi_{2}(x)+\varphi_{1}(x \pm a \hat{\mu}) \partial_{ \pm \mu} \varphi_{2}(x),
$$

where $\hat{\mu}$ denotes the unit vector along the $\mu$ direction. One might expect that, other less simple operators, instead of the simple forward/backward difference operators, could obey the usual Leibniz rule even on the lattice. This is not possible, however, due to the no-go theorem proving

\footnotetext{
${ }^{1}$ With a similar argument, we need to take the Dirac-Kähler twisted $\mathscr{N}=4$ supersymmetry in four dimensions.
} 
non-existence of such a local operator [ $[\square]$, which implies the modified Leibniz rule is unavoidable on the lattice when one concentrate on local field theories. In other words, it implies that the supercharges can't obey the normal Leibniz rule either to make the algebra (․․) hold.

In the link approach, the following modified Leibniz rule for $Q_{A}$ was assumed

$$
Q_{A}\left(\varphi_{1} \cdot \varphi_{2}\right)(x)=Q_{A} \varphi_{1}(x) \varphi_{2}(x)+(-1)^{\left|\varphi_{1}\right|} \varphi_{1}\left(x+a_{A}\right) Q_{A} \varphi_{2}(x),
$$

where $|\varphi|$ is 0 (or 1 ) when $\varphi$ is bosonic (or fermionic, respectively). This already shows that the algebra (2.1) doesn't form a Lie superalgebra in the usual sense, and "deforms" the notion of usual superalgebra. Then a natural question is whether we could treat the algebra (2.1) with the modified Leibniz rules ([2.2) and ([2.3) in a mathematically rigorous manner. We will see shortly that the answer is affirmative; the algebra in the link approach can be identified as a Hopf algebra, which assures mathematical consistency especially for the modified Leibniz rule ([2.3). Another question would immediately follows: even if the algebra itself makes sense, it is still unclear whether it corresponds to a symmetry of a local quantum field theory as usual Lie algebra does. For this, too, we will propose an affirmative answer. First, we can manage to take care of the locality with a mildly generalized statistics of fields. This statistics is in fact expressed mathematically as (trivial) braiding. Since quantum field theory for fields with such a braiding structure is known to be formulated generally as BQFT [1] , we could apply it to our case. This approach now allows us to associate our Hopf algebraic symmetry with various sets of Ward-Takahashi identities [6], showing clear relations of the Hopf algebra to a symmetry of a quantum field theory.

\section{Superalgebra on the lattice as a Hopf algebra}

Here we are going to show how the superalgebra which was originally introduced with an extra shift structure in the link approach [3] can as a whole be rigorously identified as a Hopf algebra [[]]. Before going into the detail, let us briefly summarize the Hopf algebra axioms. For a full mathematical treatment on Hopf algebra, see, for example, [ $\left[{ }^{1}\right]$.

Hopf algebra $H$ is an object which satisfies the following four axioms.

1. $H$ is an algebra, namely a vector space which has an associative product (multiplication) $\cdot: H \otimes H \rightarrow H$, where the associativity reads $\cdot \circ(\cdot \otimes \mathrm{id})=\cdot \circ(\mathrm{id} \otimes \cdot)$, and unit element $\mathbb{1}^{2}$

2. $H$ is a coalgebra, namely a vector space which has a coassociative coproduct (comultiplication) $\Delta: H \rightarrow H \otimes H$, where the coassociativity reads

$$
(\Delta \otimes \mathrm{id}) \circ \Delta=(\mathrm{id} \otimes \Delta) \circ \Delta,
$$

and counit $\varepsilon: H \rightarrow \mathbb{C}$ which satisfies the condition

$$
(\varepsilon \otimes \mathrm{id}) \circ \Delta=(\mathrm{id} \otimes \varepsilon) \circ \Delta=\mathrm{id} .
$$

3. The coproduct and counit are both algebra maps, namely,

$$
\left\{\begin{array} { l } 
{ \Delta \circ = ( \cdot \otimes \cdot ) \circ \Delta , } \\
{ \Delta ( \mathbb { 1 } ) = \mathbb { 1 } \otimes \mathbb { 1 } , }
\end{array} \quad \text { and } \quad \left\{\begin{array}{l}
\varepsilon \circ=\varepsilon \otimes \varepsilon, \\
\varepsilon(\mathbb{1})=1 .
\end{array}\right.\right.
$$

\footnotetext{
${ }^{2}$ Here id is the identity map and $\circ$ denotes composition of maps.
} 
4. $H$ has an antipode $\mathrm{S}: H \rightarrow H$, which satisfies the defining condition

$$
\cdot \circ(\mathrm{S} \otimes \mathrm{id}) \circ \Delta=\cdot \circ(\mathrm{id} \otimes \mathrm{S}) \circ \Delta=\mathbb{1} \varepsilon .
$$

It is easy to see that the superalgebra (2. of two generators, say, $Q_{A}$ and $Q_{B}$, is defined with a successive applications of $Q_{B}$ and $Q_{A}$ as in $\left(Q_{A} \cdot Q_{B}\right) \triangleright \varphi:=\left(Q_{A} \triangleright\right) \circ\left(Q_{B} \triangleright\right) \varphi,{ }^{3}$ whereas the unit operator is trivially defined as $\mathbb{1} \triangleright \varphi=\varphi$. These structures together with the "equivalence" relations, (anti-)commutation relations (메), form a universal enveloping algebra of a sort. To be specific, let us list explicit field representations for this algebra, taking the example of $\mathscr{N}=(2,2)$ Wess-Zumino model in two dimensions. The field contents are scalar bosons $\phi, \sigma$, fermions $\psi, \psi_{\mu}, \tilde{\psi}$ and auxiliary fields $\tilde{\phi}, \tilde{\sigma}$, for which the supertransformations are as follows:

$$
\begin{aligned}
& Q \phi=0, \quad Q_{\mu} \phi=\psi_{\mu}, \quad \tilde{Q} \phi=0, \\
& Q \psi_{v}=i \partial_{+v} \phi, \quad Q_{\mu} \psi_{v}=-\varepsilon_{\mu \nu} \tilde{\phi}, \quad \tilde{Q} \psi_{v}=-i \varepsilon_{v \mu} \partial_{-\mu} \phi, \\
& Q \tilde{\phi}=-i \varepsilon_{\mu v} \partial_{+\mu} \psi_{v}, \quad Q_{\mu} \tilde{\phi}=0, \quad \tilde{Q} \tilde{\phi}=i \partial_{-\mu} \psi_{\mu}, \\
& Q \sigma=-\psi, \quad Q_{\mu} \sigma=0, \quad \tilde{Q} \sigma=-\tilde{\psi}, \\
& Q \psi=0, \quad Q_{\mu} \psi=-i \partial_{+\mu} \sigma, \quad \tilde{Q} \psi=-\tilde{\sigma}, \\
& Q \tilde{\psi}=\tilde{\sigma}, \quad Q_{\mu} \tilde{\psi}=i \varepsilon_{\mu \nu} \partial_{-\nu} \sigma, \quad \tilde{Q} \tilde{\psi}=0, \\
& Q \tilde{\sigma}=0, \quad Q_{\mu} \tilde{\sigma}=i \varepsilon_{\mu \nu} \partial_{-\nu} \psi+i \partial_{+\mu} \tilde{\psi}, \quad \tilde{Q} \tilde{\sigma}=0 .
\end{aligned}
$$

What is more important is the coproduct structure. It amounts to specifying the action of an operator, say $Q_{A}$, on a product of fields $\varphi_{1} \cdot \varphi_{2}=: m\left(\varphi_{1} \otimes \varphi_{2}\right)$ as in

$$
Q_{A} \triangleright\left(\varphi_{1} \cdot \varphi_{2}\right)=m\left(\Delta\left(Q_{A}\right) \triangleright\left(\varphi_{1} \otimes \varphi_{2}\right)\right) .
$$

Thus determining the coproduct structure is nothing but to specifying the Leibniz rule. For instance, the modified Leibniz rule ([2.3]) is essentially equivalent to the coproduct formula

$$
\Delta\left(Q_{A}\right)=Q_{A} \otimes \mathbb{1}+(-1)^{\mathscr{F}} \cdot T_{a_{A}} \otimes Q_{A},
$$

where $(-1)^{\mathscr{F}}$ just gives factor +1 (or -1 ) when applied on a bosonic (or fermionic, resp.) field: $(-1)^{\mathscr{F}} \triangleright \varphi= \pm \varphi$, and $T_{a_{A}}$ is the shift operator: $\left(T_{a_{A}} \triangleright \varphi\right)(x):=\varphi\left(x+a_{A}\right)$. Note in passing that these operators would satisfy the trivial (anti-)commutation relations

$$
\left[Q_{A}, T_{b}\right]=\left[P_{\mu}, T_{b}\right]=\left[T_{b}, T_{c}\right]=0, \quad\left\{Q_{A},(-1)^{\mathscr{F}}\right\}=\left[P_{A},(-1)^{\mathscr{F}}\right]=\left[T_{b},(-1)^{\mathscr{F}}\right]=0 .
$$

We can determine the coproduct for $\partial_{ \pm \mu}, T_{b}$ and $(-1)^{\mathscr{F}}$ by the identifications similar to (B.6), which result in the following formulae:

$$
\Delta\left(\partial_{ \pm \mu}\right)=\partial_{ \pm \mu} \otimes \mathbb{1}+T_{ \pm a \hat{\mu}} \otimes \partial_{ \pm \mu}, \quad \Delta\left(T_{b}\right)=T_{b} \otimes T_{b}, \quad \Delta\left((-1)^{\mathscr{F}}\right)=(-1)^{\mathscr{F}} \otimes(-1)^{\mathscr{F}}
$$

We can confirm ourselves, by straightforward calculations, that these prescriptions indeed obey the coassociativity condition (B. Cl). Notice that the coassociativity condition assures the uniqueness of

\footnotetext{
${ }^{3}$ The "action" of a generator $a$ on a field $\varphi$ is denoted as $a \triangleright \varphi$.
} 
the action of an operator on a product of three or more fields. For instance, since by the associativity $\varphi_{1} \cdot \varphi_{2} \cdot \varphi_{3}=\left(\varphi_{1} \cdot \varphi_{2}\right) \cdot \varphi_{3}=\varphi_{1} \cdot\left(\varphi_{2} \cdot \varphi_{3}\right)$, we need $Q_{A} \triangleright\left(\varphi_{1} \cdot \varphi_{2} \cdot \varphi_{3}\right)=Q_{A} \triangleright\left(\left(\varphi_{1} \cdot \varphi_{2}\right) \cdot \varphi_{3}\right)=$ $Q_{A} \triangleright\left(\varphi_{1} \cdot\left(\varphi_{2} \cdot \varphi_{3}\right)\right)$. This is equivalent to $(\Delta \otimes \mathrm{id}) \circ \Delta\left(Q_{A}\right)=(\operatorname{id} \otimes \Delta) \circ \Delta\left(Q_{A}\right)$, which is the coassociativity condition for the operator $Q_{A}$. Similar arguments of course hold for other operators.

The coproduct structure thus determines how operators act on products of fields. Note, however, that any field $\varphi$ can be considered as a product $\varphi=\mathbf{1} \cdot \varphi=\varphi \cdot \mathbf{1}$ with the constant field 1. Accordingly, when the operator, say, $Q_{A}$ acts on $\varphi$, it must satisfy the consistency condition $Q_{A} \triangleright \varphi=Q_{A} \triangleright(\mathbf{1} \cdot \varphi)=Q_{A} \triangleright(\boldsymbol{\varphi} \cdot \mathbf{1})$. In order to state this more generally, let us define the counit map by

$$
Q_{A} \triangleright \mathbf{1} \equiv \varepsilon\left(Q_{A}\right) \mathbf{1},
$$

so that the counit gives the trivial representation. The consistency above is now written as id = $(\varepsilon \otimes \mathrm{id}) \circ \Delta=(\mathrm{id} \otimes \varepsilon) \circ \Delta$, which is what we listed before (B.2). The explicit formulae (B.7) and (B.9) now allow us to specify the counit of operators which satisfies this condition as follows:

$$
\varepsilon\left(Q_{A}\right)=0, \quad \varepsilon\left(P_{\mu}\right)=0, \quad \varepsilon\left(T_{b}\right)=1, \quad \varepsilon\left((-1)^{\mathscr{F}}\right)=1 .
$$

Coproduct and counit for a product of operators can be calculated through the algebra-map conditions (B.3)). We emphasize that this property is important since it also assures the explicit formulae (B.7), (B.9) and (B.Tl) are indeed compatible with the algebraic relations (2..W).

We introduce one more object, the antipode. It essentially gives the "inverse" of operators, uniquely determined by the relation (B.4). From the explicit formulae (B.7), (B.9) and (B.DI), we find the following formulae

$$
\mathrm{S}\left(Q_{A}\right)=-(-1)^{\mathscr{F}} \cdot Q_{A}, \quad \mathrm{~S}\left(P_{\mu}\right)=-P_{\mu}, \quad \mathrm{S}\left(T_{b}\right)=T_{b}^{-1}, \quad \mathrm{~S}\left((-1)^{\mathscr{F}}\right)=(-1)^{\mathscr{F}} .
$$

We can show by the condition (3.4) that the antipode is anti-algebraic, namely, $\mathrm{S} \circ \cdot=\cdot \circ \tau \circ(\mathrm{S} \otimes \mathbf{S})$ and $\mathbf{S}(\mathbb{1})=\mathbb{1}$, where $\tau$ is the transposition $\tau(a \otimes b):=b \otimes a$. This is again consistent with the relation (‥J), as seen with the explicit formulae (B.7), (B.9) and (B.J1). We can also derive that the antipode should satisfy anti-coalgebraic nature as in $(S \otimes S) \circ \Delta=\tau \circ \Delta \circ S$ and $\varepsilon \circ S=\varepsilon$, which are also found to be compatible to the explicit formulae.

\section{Statistics on the lattice as a braiding}

Our next task is to consider field-product representations of the Hopf algebraic supersymmetry. We first emphasize here that a Hopf algebra in general has a noncommutative representation. In the current application, a noncommutative representation would naturally lead to a noncommutative field theory, which would then be nonlocal. In fact, we could avoid this noncommutativity or nonlocality, systematically taking product representations which are almost commutative, or, in other words, commutative up to a mildly generalized statistics. ${ }^{4}$

We illustrate more concretely how this is possible with the previous example of $\mathscr{N}=(2,2)$ Wess-Zumino model in two dimensions. For scalars $\phi, \sigma$, supertransformations with respect to

\footnotetext{
${ }^{4}$ Our Hopf algebra has a simple structure, (quasi)triangularity, which allows such an almost commutative representation.
} 
$Q_{\mu}$ are given as $Q_{\mu} \phi=\psi_{\mu}, Q_{\mu} \sigma=0$ (see (B.5)). Let us assume that the scalars be commutative: $\phi(x) \cdot \sigma(x)=\sigma(x) \cdot \phi(x)$. The point is, once we set this assumption, we could deduce from the supertransformations (B.5) the statistics for the other fields in a manner totally consistent with the Hopf algebra structures. To see this, we calculate $Q_{\mu}(\phi(x) \cdot \sigma(x))=Q_{\mu}(\sigma(x) \cdot \phi(x))$, so that, with the use of the coproduct formula (B.]), we have $\psi_{\mu}(x) \cdot \sigma(x)=\sigma\left(x+a_{\mu}\right) \cdot \psi_{\mu}(x)$. This shows that the fermion $\psi_{\mu}$ is commutative with the boson $\sigma$ up to the shift of argument. Similar calculations lead to that $\psi_{\mu}$ is (anti-)commutative with any other fields up to the same amount of shift of argument. We can in fact generalize this statement as follows

$$
\Psi\left(\varphi_{A_{0} \cdots A_{p}}(x) \otimes \varphi_{B_{0} \cdots B_{q}}^{\prime}(y)\right)=(-1)^{p q} \varphi_{B_{0} \cdots B_{q}}^{\prime}\left(y+\sum_{i=1}^{p} a_{A_{i}}\right) \otimes \varphi_{A_{0} \cdots A_{p}}\left(x-\sum_{i=1}^{q} a_{B_{i}}\right),
$$

where $\Psi$ represents the exchange of the order of fields in a tensor product, called (trivial) braid, and $\varphi_{A_{0} \cdots A_{p}}:=Q_{A_{p}} \cdots Q_{A_{1}} \varphi_{A_{0}}$, with $\varphi_{A_{0}}$ denoting scalars $\phi$ or $\sigma$.

With the mildly generalized statistics (4.]), the ordering ambiguity claimed in [Q] no longer appears. ${ }^{5}$ Notice also that we might understand this statistics property in terms of a grading structure for each field and symmetry operator which is determined corresponding to the indices $A_{i}$ and $B_{i}$ in the formula (4. (ل) Then in particular the difference operators $\partial_{ \pm \mu}$ as well must have the grading structure, which is difficult to express explicitly. We will come to this point in the conclusion.

\section{Supersymmetric lattice field theory as BQFT}

Quantum field theory for fields with generalized statistics, or braiding, can be generally formulated as braided quantum field theory (BQFT) at least perturbatively [ $[$ ]. We can thus apply the formalism into our approach to construct a perturbative lattice field theory. Here we just sketch the outline of the formulation. The theory is quantized through path integral formalism

$$
Z=\int e^{-S}, \quad\left\langle\varphi_{1} \cdots \varphi_{n}\right\rangle=\frac{1}{Z} \int \varphi_{1} \cdots \varphi_{n} e^{-S}, \quad \int \frac{\delta}{\delta \varphi(x)}=0
$$

for a classical action $S$. The last equation formally defines the path integral, for which the functional derivative is assumed to obey the deformed Leibniz rule

$$
\frac{\delta}{\delta \varphi(x)}\left(\varphi_{1} \cdot \varphi_{2}\right)=\frac{\delta}{\delta \varphi(x)} \varphi_{1} \cdot \varphi_{2}+(-1)^{|\varphi|\left|\varphi_{1}\right|} T_{\varphi}^{-1} \varphi_{1} \cdot \frac{\delta}{\delta \varphi(x)} \varphi_{2}
$$

The formal expression is enough to derive perturbative Wick's theorem with appropriate statistics, which allows to compute arbitrary correlation functions in terms of propagators determined by the specific form of the classical action. Now the classical Hopf algebraic supersymmetry is expressed by $Q_{A} \triangleright S=0$. At the quantum level, this leads to various sets of Ward-Takahashi identities of the form [䧃]

$$
Q_{A} \triangleright\left\langle\varphi_{1} \cdots \varphi_{n}\right\rangle=\varepsilon\left(Q_{A}\right)\left\langle\varphi_{1} \cdots \varphi_{n}\right\rangle=0
$$

\footnotetext{
${ }^{5}$ Another difficulty raised there in the case of gauge theory needs further investigations.
} 


\section{Conclusion}

In this article, we presented a formulation of lattice supersymmetry with the machinery of Hopf algebra and BQFT, based on the previously proposed formulation, the link approach. We showed explicitly that superalgebra on a lattice can be identified as a Hopf algebra, where the modified or deformed Leibniz rules invented in the original link approach are now incorporated as the coproduct structure in the Hopf algebra. Fields, as representations of the Hopf algebraic symmetry, would in general be noncommutative, thus the corresponding field theory would be nonlocal. This noncommutativity, however, could be reduced to the commutativity up to a lattice-deformed statistics in a manner consistent with the Hopf algebraic superalgebra. The difficulty claimed as ordering ambiguity against the original link approach is now solved thanks to this deformed statistics. We then applied the formalism of BQFT to construct a quantum field theory for such a generalized statistics, which allows us to derive Ward-Takahashi identities associated with the Hopf algebraic supersymmetry at least perturbatively.

In this formulation, fields and symmetry generators could be interpreted to have grading structures corresponding to the deformed statistics of fields. In particular, "momentum" operator, the difference operators on the lattice, should have nontrivial grading. In order to compute arbitrary correlation functions, especially when including loop corrections, we need an explicit representation of the graded difference operators. Such a representation might be unnecessary for the computations of physical observables. Another issue is that this construction at the moment is limited only on a formal and perturbative level, and it is not yet clear whether it can lead to nonperturbative formulation as a lattice field theory. Gauge theory extension is missing as well. These issues are for the future works.

\section{References}

[1] S. Catterall, D. B. Kaplan and M. Unsal, arXiv:0903.4881 [hep-lat].

[2] A. D'Adda, N. Kawamoto and J. Saito, arXiv:0907.4137 [hep-th].

[3] A. D’Adda, I. Kanamori, N. Kawamoto and K. Nagata, Nucl. Phys. B 707 (2005) 100 [arXiv:hep-lat/0406029]; Phys. Lett. B 633 (2006) 645 [arXiv:hep-lat/0507029]; Nucl. Phys. B 798, 168 (2008) [arXiv:0707.3533 [hep-lat]].

[4] S. Arianos, A. D'Adda, A. Feo, N. Kawamoto and J. Saito, Int. J. Mod. Phys. A (to be published) [arXiv:0806.0686 [hep-lat]].

[5] R. Oeckl, Commun. Math. Phys. 217 (2001) 451 [arXiv:hep-th/9906225].

[6] Y. Sasai and N. Sasakura, Prog. Theor. Phys. 118 (2007) 785 [arXiv:0704.0822 [hep-th]].

[7] M. Kato, M. Sakamoto and H. So, JHEP 0805, 057 (2008) [arXiv:0803.3121 [hep-lat]].

[8] S. Majid, Cambridge, UK: Univ. Pr. (1995) 607 p.

[9] F. Bruckmann and M. de Kok, Phys. Rev. D 73 (2006) 074511 [arXiv:hep-lat/0603003]; F. Bruckmann, S. Catterall, and M. de Kok, Phys. Rev. D 75 (2007) 045016 [arXiv:hep-lat/0611001]. 